\title{
Amount of Calcium Elution and Eroded Lesion Depth in Bovine Enamel Derived from Single Short Time Immersion in Carbonated Soft Drink in Vitro
}

\author{
Koji Watanabe1, Toshiko Tanaka², Kenshi Maki³, Hideaki Nakashima², \\ Shigeru Watanabe ${ }^{1}$ \\ ${ }^{1}$ Division of Pediatric Dentistry, Department of Development and Fostering, Meikai University School of \\ Dentistry, Saitama, Japan \\ ${ }^{2}$ Division of Surgery, Department of Health Promotion, Kyushu Dental University, Fukuoka, Japan \\ ${ }^{3}$ Division of Developmental Stomatognathic Function Science, Department of Health Promotion, Kyushu Dental \\ University, Fukuoka, Japan \\ Email: k-watanabe@dent.meikai.ac.jp, tana-tos@kyu-dent.ac.jp, k-maki@kyu-dent.ac.jp, \\ r09nakashima2@fa.kyu-dent.ac.jp, shigeru@dent.meikai.ac.jp
}

Received 27 February 2015; accepted 17 March 2015; published 19 March 2015

Copyright (C) 2015 by authors and Scientific Research Publishing Inc.

This work is licensed under the Creative Commons Attribution International License (CC BY). http://creativecommons.org/licenses/by/4.0/

(c) (i) Open Access

\section{Abstract}

Erosion is one of serious oral health problems among Japanese children. Some dentists argue that brushing just after food/drink intake induces enamel abrasion. Objectives of this study were to evaluate amount of calcium elution from bovine enamel due to single and short immersion into carbonated soft drink, to calculate depth of the eroded lesion, and to determine when to brush teeth after carbonated soft drink intake from the view point of preventing enamel abrasion. Four enamel specimens were made from each of eight bovine teeth. The specimens were covered by quick-cure resin except for enamel surfaces. The four specimens from each bovine tooth were classified into three, six, nine, and 12 minutes immersion (IM3, IM6, IM9, and IM12) groups and immersed separately in five $\mathrm{mL}$ of carbonated soft drink. After the immersion, the calcium concentration of the original drink and the drink samples were evaluated using atomic absorption spectrophotometry. The dimension of each enamel specimen was calculated using a planimeter. The amount of eluted calcium per unit area of the enamel specimen into each drink sample was obtained. The depth of the demineralized lesion was obtained by dividing the amount of calcium elution per unit area by the concentration of calcium in enamel and the specific gravity of enamel. The lesion depth of the IM3 group was significantly lower than those in the IM6, IM9, and IM12 groups. The mean lesion depth in the IM12 group which showed the deepest lesion depth was 0.21 
$\mu \mathrm{m}$. As conclusions, the erosive lesion depth due to intake of carbonated soft drink was much shallower than remineralized enamel surface of a white spot lesion which can be repaired in plaque fluid in a short time, suggesting such erosion hardly causes tooth wear, hence it was suggested that brushing teeth just after the intake was recommended.

\title{
Keywords
}

\author{
Erosion, Bovine Enamel, Carbonated Soft Drink, Calcium
}

\section{Introduction}

As caries prevalence decreases, erosion is becoming one of the important oral health problems in some countries, including Japan. Caries is initiated as demineralization due to bacterially-derived acid production. On the other hand, erosion is initiated as demineralization due to non-bacterially-derived acid exposure such as in bulimia, gastric acid reflex, and frequent acidic food intake [1]. In our daily life, intake of acidic drinks is a common event that causes erosion [2]-[5]. Brushing eroded teeth is considered to be one of the mechanisms of enamel abrasion. To date, ways to prevent the abrasion of eroded enamel due to brushing have been studied, by immersing enamel samples cyclically in an acidic solution and then a remineralization solution several times [6][9]. Since the protocols of the previous studies simulate frequent or long term acid exposure, some dentists, parents, and teachers consider that brushing teeth after acid drink intake always induces enamel abrasion. Attin et al. [10] also recommended brushing teeth one hour after acid explosion to protect dental hard tissue from increased loss. Enamel erosion progresses layer by layer based on the exposure time and degree of saturation with respect to hydroxyapatite of the solution surrounding the enamel [11]. In the present study, the amount of calcium (Ca) elution per unit area of bovine enamel through single and short immersion into carbonated soft drink was measured and the depth of the eroded lesion was calculated from the measured value, Ca composition and specific gravity of hydroxyapatite crystals. We have then discussed when to brush teeth to prevent tooth abrasion after single and short exposure to carbonated soft drink in healthy enamel of normal people.

\section{Materials and Methods}

\subsection{Preparation of Enamel Specimens}

Bovine teeth were purchased from a meat trader before the teeth were discarded. The enamel surface of the eight bovine teeth were ground flat and polished by 400-, 600-, and 800-grit silicon carbide abrasive papers to remove extraneous matter from the surface, resulting in enamel removal of the very surface zone where is less acid-soluble because of the higher distribution of fluoride compared with newly exposed surface zone. Four enamel specimens measuring four-by-four mm with a thickness of five mm were made from each of eight bovine labial surfaces. The specimens were embedded in quick cure resin (GC CORPORATION, Tokyo, Japan), exposing the enamel surfaces. When the enamel specimens were embedded, the enamel surface was covered by vinyl tape which was cut into the same shape as the enamel surface so as not to cover the enamel surface by quick cure resin. The vinyl tape was removed after the resin cured and then the dimensions of the vinyl tape were measured using a Planix 10S Digital Planimeter (Marble, Sydney, Australia).

\subsection{Immersion of the Enamel Specimens}

The four embedded enamel samples from each bovine tooth were ultrasonically washed with ultrapure water three times for five minutes each time, dried at room temperature, and immersed separately in five $\mathrm{mL}$ of carbonated soft drink (MITSUYA CIDER; ASAHI SOFT DRINKS CO., Ltd., Tokyo, Japan) for three, six, nine, and 12 minutes, respectively. Each enamel sample was kept in a sterilized polypropylene tube (Falcon ${ }^{\circledR}$; Becton, Dickinson and Company Japan, Tokyo, Japan) containing $5 \mathrm{~mL}$ of carbonated soft drink and shaken at $37^{\circ} \mathrm{C}$ throughout the immersion. In three, six, nine, and 12 minutes of immersion, enamel samples of the relevant immersion time were removed from the drink. 


\subsection{Measurement of Ca Elution}

The Ca concentration of the original carbonated soft drink and drink samples in each group was measured using atomic absorption spectrophotometry (A Analyst 100; PerkinElmer Japan, Co., Ltd., Tokyo, Japan). The drink sample was atomized by aspiration in an air-acetylene flame after three-time dilution with $0.1 \mathrm{~mol} / \mathrm{L} \mathrm{HNO}_{3}$. Each element level was determined by the calibration curve method using standard solutions for atomic absorption spectrophotometry. The samples and standard solutions were diluted with $0.1 \mathrm{~mol} / \mathrm{L} \mathrm{HNO}$ as necessary. All chemicals were of the highest available purity. Each sample was measured in duplicate. The standard curve was recalibrated every ten samples. The concentration of $\mathrm{Ca}$ was calculated as $\mathrm{mg} / \mathrm{L}$ including the originally contained $\mathrm{Ca}$ and eluted $\mathrm{Ca}$ from the enamel surface. The amount of eluted $\mathrm{Ca}$ into each drink sample per unit area was obtained by the difference between the measured value in each drink sample and the originally contained amount of Ca.

\subsection{Calculation of the Lesion Depth}

Depth of the demineralized lesion was obtained by dividing the amount of calcium elution into the drink sample by the dimension of the enamel surface, the percentage calcium in hydroxyapatite crystals $(0.37)$ and the specific gravity of hydroxyapatite crystals (2.95) (Figure 1).

\subsection{Statistical Analysis}

The significance of the difference among the groups was analyzed by one-way ANOVA and Tukey's HSD. $p<$ 0.05 was considered significant.

\section{Results}

Values shown in the text, Tables and Figures are expressed as mean \pm standard error (S.E.). Carbonated soft drink originally contained $6.97 \pm 0.02 \mathrm{mg} / \mathrm{L}$ of Ca. As shown in Table 1, the amount of Ca elution per unit area of enamel surface in the IM3, IM6, IM9 and IM 12 was $0.06 \pm 0.02,0.18 \pm 0.03,0.17 \pm 0.03$ and $0.23 \pm 0.04$ $\mu \mathrm{g} / \mathrm{mm}^{2}$, respectively. Ca elution in the IM3 group was significantly lower than that of the IM6 $(p<0.05)$, IM9 $(p<0.05)$ and IM12 $(p<0.01)$ groups. In addition, the lesion depth in the IM3 was also significantly lower than that of IM6 ( $p<0.05)$, IM9 $(p<0.05)$ and IM12 $(p<0.01)$ groups (Figure 2$)$.

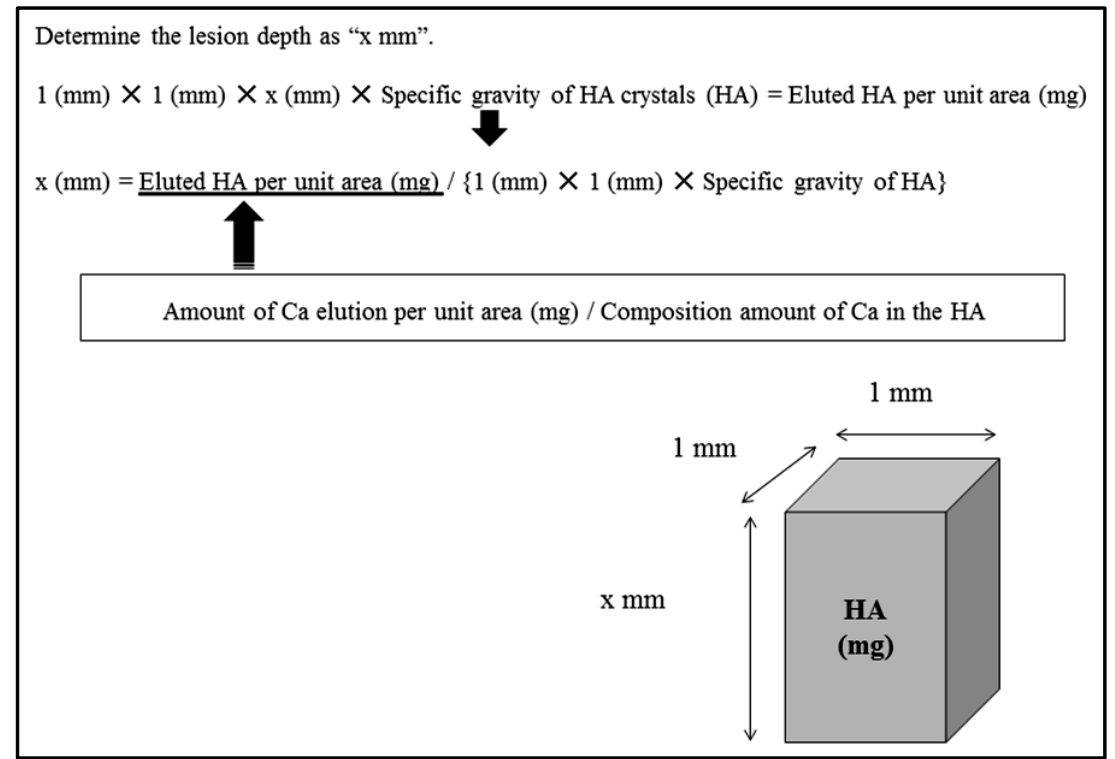

Figure 1. Calculation of lesion depth of erosion. Erosive lesion depth was calculated by dividing eluted HA per unit area into specific gravity of HA crystals. The eluted HA was obtained by dividing amount of Ca elution per unit area into composition amount of $\mathrm{Ca}$ in the HA crystals. 
Table 1. Amount of Ca elution per unit area of enamel surface.

\begin{tabular}{ccc}
\hline Group & \multicolumn{1}{c}{ Ca elution $\left(\mu \mathrm{g} / \mathrm{mm}^{2}\right)$} & \\
\hline IM3 & $0.06 \pm 0.02$ & \\
IM6 & $0.18 \pm 0.03$ & \\
IM9 & $0.17 \pm 0.03$ & \\
IM12 & \\
\hline
\end{tabular}

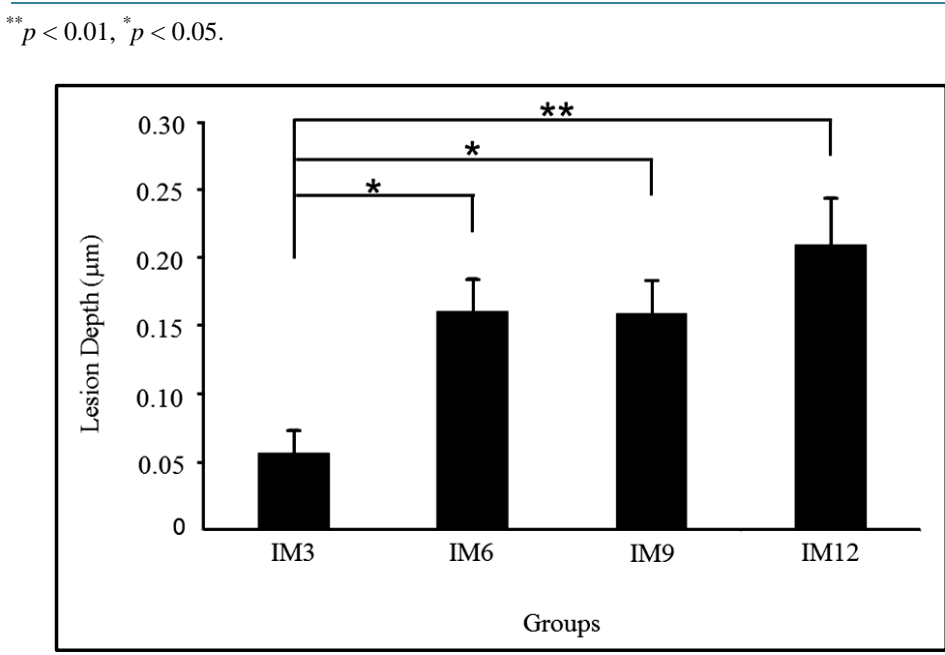

Figure 2. Lesion depth in each group. Lesion depth in the IM3 group was significantly lower than that of IM6, IM9 and IM12 groups. ${ }^{* *} p<0.01,{ }^{*} p<0.05$.

\section{Discussion}

Ca is one of the major minerals contained in enamel and hence is used as one of the important parameters of demineralization [12] [13]. In the present study, the amount of eluted Ca per unit area of the enamel surface and the lesion depth were proportional to the immersion time as mentioned in the result section and Figure 2 indicating that erosion progressed layer by layer. This was supported by previously published literature [11].

There are two kinds of pathological demineralization; a caries lesion is due to bacterially-derived acid production, while erosion is due to non-bacterially-derived acid. In caries lesions, demineralization begins on the enamel surface where it is covered by biofilm, but most mineral loss occurs in a subsurface location. The demineralized lesion may be remineralized by re-charging minerals based on the concentration gradient from plaque fluid, which present in the biofilm on the enamel surface. On the other hand, in erosion, demineralization begins on the enamel surface and progresses layer by layer. In the present study, the lesion depth of erosion could be calculated by the amount of eluted Ca from a unit area of enamel surface since the Ca could be considered to be eluted layer by layer from the enamel surface and lesion depth of erosion was found to have ranged up to 0.21 $\mu \mathrm{m}$. In a previous study, it was reported that a short acid exposure leads to a fragile demineralized layer of about $0.5 \mu \mathrm{m}$, supporting our result [14].

Huang et al. [15] reported in their in vitro study analyzing white spot lesions in human enamel by X-ray micro-tomography that the depth of a white spot lesion in natural human enamel ranged about $50-600 \mu \mathrm{m}$. Their report can be interpreted as showing that the initially demineralized enamel was repaired in plaque fluid up to 50 $\mu \mathrm{m}$ in depth from the enamel surface. Moreover, Jensdottir et al. [16] reported in their in vivo study on wholemouth saliva composition of males that sucking acidic candy that increased salivary flow rate and buffer capacity of stimulated saliva had protective effects against erosion, suggesting that stimulated saliva has potential to repair demineralized enamel in the early stage of erosion. Considering these previous reports, it should be noted that while experiment conditions were different among studies, the present study suggested that the potential of saliva was enough to repair demineralized enamel over $0.21 \mu \mathrm{m}$ in depth due to a single carbonated soft drink intake. 
In previous in vitro [6]-[9] [17]-[19] and in vivo [20] studies evaluating de- and remineralization, enamel specimens were immersed in the demineralization solution for extremely long terms or more frequently, compared with ordinary time or frequency of consuming soft drinks in our daily life; in the oral cavity, acid exposure is usually short [21]. Such experimental conditions are based on the fact that erosion and abrasion become serious by long term or frequent acid food/drink intake; however, not all people have such a habit.

Voronets and Lussi [22] evaluated the thickness of demineralized human enamel removed by brushing after immersion in orange juice and reported that $268 \mathrm{~nm}$ of enamel was removed. In their study, demineralization was carried out by immersing human enamel into $50 \mathrm{~mL}$ of orange juice for three minutes. The thickness of removed enamel by brushing after three minutes of immersion into orange juice was markedly higher than the lesion depth obtained in the present study; however, the degree of demineralization which increases the porosity of enamel is affected by various factors such as temperature, flow rate, degree of saturation, presence of inhibitors, buffering, $\mathrm{pH}$, fluoride [23]. The carbonated soft drink used in the present study as a demineralization solution originally contained $6.97 \pm 0.02 \mathrm{mg} / \mathrm{L}$ of Ca. Ca is reported to be one of the protective factors against erosion when added as one of the ingredients of food [24], which could reduce the lesion depth in this study.

As reported in previous studies, eroded enamel is extraordinarily sensitive to mechanical impacts such as tooth brushing performed immediately after demineralization [25] [26]. Attin et al. [27] reported in their in vitro study on the time to improve the abrasion resistance of previously eroded bovine enamel that abrasion resistance of eroded enamel in in vitro conditions continuously increases with remineralization time. In the previous study, a one-hour interval of remineralization between a meal and tooth brushing was recommended under in vitro conditions; however, the authors declared that in an every-day situation it is not feasible to allow such a long interval to elapse before tooth brushing. Certainly, under in vitro conditions, enamel specimens were exposed to only acid. In contrast, under in vivo conditions, saliva is continuously secreted during food/drink intake resulting in buffering acid existing in the oral cavity with possible repair of eroded enamel surfaces. Moreover, it has to be taken into consideration that there are biofilms on the enamel surfaces in a real oral cavity. If a person does not brush his/her teeth for an hour after meal, bacterial-derived acid production occurs resulting in caries lesion. Plaque $\mathrm{pH}$ falls dramatically below the critical $\mathrm{pH}$ within a few minutes after sucrose intake [28]. Opposing the studies which argued that eroded enamel was sensitive to mechanical stress or that the interval between food/ drink intake and tooth brushing was favorable, Ganss et al. concluded that a waiting period between acid exposure and tooth brushing had only a minor effect on the prevention of toothbrush abrasion of acid-softened enamel surface and that topical fluoride treatment could represent an effective therapeutic approach in their in situ study [29]. The present study also indicated that a single intake of carbonated soft drink for 12 minutes induced $0.21 \mu \mathrm{m}$ of erosion depth which could be repaired by saliva, supporting the previous study.

As mentioned in the Introduction section, Attin et al. [10] discussed in their in vivo study on brushing abrasion of softened human dentin that at least one hour interval is needed for eroded enamel to be remineralized, hence recommended to refrain from tooth brushing after a meal for one hour to protect eroded dental hard tissues from increased loss. However, focusing on enamel, Wiegand et al. [14] reported in their study on susceptibility of eroded enamel to mechanical wear comparing tooth brushing and ultrasonication that the susceptibility of the demineralized enamel depended on the type of mechanical force applied. Wiegand et al. [21] reported in another in vitro study using human enamel that enamel loss of acid-softened samples increases with increasing brushing strokes, but individual threshold of susceptibility to tooth brushing abrasion was reached for each brushing load. Previously published literature [30] also mentioned that a brushing load between 100 and $200 \mathrm{~g}$ is considered not to damage the tooth tissues. These previous reports suggested that only excess load and stroke of tooth brushing result in enamel abrasion.

\section{Conclusions}

1) Amount of Ca elution per unit area of bovine enamel surface due to single immersion into carbonated soft drink for 12 minutes was found to be $0.23 \mu \mathrm{g} / \mathrm{mm}^{2}$.

2) Demineralized lesion depth was calculated based on the amount of eluted Ca and determined to be 0.21 $\mu \mathrm{m}$.

3) The lesion depth is within the enamel surface that is remineralized by plaque fluid in the process of subsurface lesion formation, suggesting that the effect of remineralization by saliva reaches the eroded lesion enough and thus tooth wear can be hardly induced due to the erosion. 
4) It is suggested that demineralization of enamel surface due to appropriate single intake of carbonated soft drink hardly induces abrasion, and thus they should brush teeth as soon as possible after intake.

5) It is suggested that brushing teeth in a proper way as soon as possible is recommended to prevent pathological enamel demineralization.

6) It is suggested that food/drink which contains protective ingredients against erosion, such as Ca, should be consumed.

\section{Acknowledgements}

The authors acknowledge Dr. Colin Dawes, Professor Emeritus, Department of Oral Biology, Faculty of Dentistry, University of Manitoba for giving advices to improve English grammar and format of our manuscript.

\section{References}

[1] Khamverdi, Z., Vahedi, M., Abdollahzadeh, S. and Ghambari, M.H. (2013) Effect of a Common Diet and Regular Beverage on Enamel Erosion in various Temperatures: An in-Vitro Study. Journal of Dentistry of Tehran University of Medical Sciences, 10, 411-416.

[2] Borjian, A., Ferrari, C.C., Anouf, A. and Touyz, L.Z. (2010) Pop-Cola Acids and Tooth Erosion: An in Vitro, in Vivo, Electron-Microscopic, and Clinical Report. International Journal of Dentistry, 2010, 957842. http://dx.doi.org/10.1155/2010/957842

[3] Torres, C.P., Chinelatti, M.A., Gomes-Silva, J.M., Rizóli, F.A., Oliveira, M.A., Palma-Dibb, R.G. and Borsatto, M.C. (2010) Surface and Subsurface Erosion of Primary Enamel by Acid Beverages over Time. Brazilian Dental Journal, 21, 337-345. http://dx.doi.org/10.1590/S0103-64402010000400009

[4] Casas-Apayco, L.C., Dreibi, V.M., Hipólito, A.C., Graeff, M.S., Rios, D., Magalhães, A.C., Buzalaf, M.A. and Wang, L. (2014) Erosivecola-Based Drinks Affect the Bonding to Enamel Surface: An in Vitro Study. Journal of Applied Oral Science, 22, 434-441.

[5] Ulusoy, C, Müjdeci, A. and Gökay, O. (2009) The Effect of Herbal Teas on the Shear Bond Strength of Orthodontic Brackets. The European Journal of Orthodontics, 31, 385-389. http://dx.doi.org/10.1093/ejo/cjn129

[6] Austin, R.S., Stenhagen, K.S., Hove, L.H., Dunne, S., Moazzez, R., Bartlett, D.W. and Tveit, A.B. (2011) A Qualitative and Quantitative Investigation into the Effect of Fluoride Formulations on Enamel Erosion and Erosion-Abrasion in Vitro. Journal of Dentistry, 39, 648-655. http://dx.doi.org/10.1016/j.jdent.2011.07.006

[7] Carvalho, T.S. and Lussi, A. (2014) Combined Effect of a Fluoride-, Stannous- and Chitosan-Containing Toothpaste and Stannous-Containing Rinse on the Prevention of Initial Enamel Erosion-Abrasion. Journal of Dentistry, 42, 450459. http://dx.doi.org/10.1016/j.jdent.2014.01.004

[8] Pancote, L.P., Manarelli, M.M., Danelon, M. and Delbem, A.C. (2014) Effect of Fluoride Gels Supplemented with Sodium Trimetaphosphate on Enamel Erosion and Abrasion: In Vitro Study. Archives of Oral Biology, 59, 336-340. http://dx.doi.org/10.1016/j.archoralbio.2013.12.007

[9] Wiegand, A., Schneider, S., Sener, B., Roos, M. and Attin, T. (2014) Stability against Brushing Abrasion and the Erosion-Protective Effect of Different Fluoride Compounds. Caries Research, 48, 154-162. http://dx.doi.org/10.1159/000353143

[10] Attin, T., Siegel, S., Buchalla, W., Lennon, A.M., Hannig, C. and Becker, K. (2004) Brushing Abrasion of Softened and Remineralised Dentin: An in Situ Study. Caries Research, 38, 62-66. http://dx.doi.org/10.1159/000073922

[11] Larsen, M.J. (2008) Erosion of the Teeth. In: Fejerskov, O. and Kidd, E., Eds., Dental Caries: The Disease and Its Clinical Management, Blackwell Munksgaard, Oxford, 233-247.

[12] Meckelburg, N., Pinto, K.C., Farah, A., Iorio, N.L., Pierro, V.S., Dos Santos, K.R., Maia, L.C. and Antonio, A.G. (2014) Antibacterial Effect of Coffee: Calcium Concentration in a Culture Containing Teeth/Biofilm Exposed to Coffea canephora Aqueous Extract. Letters in Applied Microbiology, 59, 342-347. http://dx.doi.org/10.1111/lam.12281

[13] Yetkiner, E., Wegehaupt, F.J., Attin, R., Wiegand, A. and Attin, T. (2014) Stability of Two Resin Combinations Used as Sealants against Toothbrush Abrasion and Acid Challenge in Vitro. Acta Odontologica Scandinavica, 72, 825-830.

[14] Wiegand, A., Wegehaupt, F., Werner, C. and Attin, T. (2007) Susceptibility of Acid-Softened Enamel to Mechanical Wear-Ultrasonication versus Toothbrushing Abrasion. Caries Research, 41, 56-60. http://dx.doi.org/10.1159/000096106

[15] Huang, T.T., Jones, A.S., He, L.H., Darendeliler, M.A. and Swain, M.V. (2007) Characterisation of Enamel White Spot Lesions Using X-Ray Micro-Tomography. Journal of Dentistry, 35, 737-743.

http://dx.doi.org/10.1016/j.jdent.2007.06.001 
[16] Jensdottir, T., Nauntofte, B., Buchwald, C. and Bardow, A. (2005) Effects of Sucking Acidic Candy on Whole-Mouth Saliva Composition. Caries Research, 39, 468-474. http://dx.doi.org/10.1159/000088181

[17] Vongsavan, K., Surarit, R. and Rirattanapong, P. (2014) The Combined Effect of Xylitol and Fluoride in Varnish on Bovine Teeth Surface Microhardness. Southeast Asian Journal of Tropical Medicine and Public Health, 45, 505-510.

[18] Bakry, A.S., Marghalani, H.Y., Amin, O.A. and Tagami, J. (2014) The Effect of a Bioglass Paste on Enamel Exposed to Erosive Challenge. Journal of Dentistry, 42, 1458-1463. http://dx.doi.org/10.1016/j.jdent.2014.05.014

[19] Lata, S., Varghese, N.O. and Varughese, J.M. (2010) Remineralization Potential of Fluoride and Amorphous Calcium Phosphate-Casein Phospho Peptide on Enamel Lesions: An in Vitro Comparative Evaluation. Journal of Conservative Dentistry, 13, 42-46. http://dx.doi.org/10.4103/0972-0707.62634

[20] Sullivan, R., Rege, A., Corby, P., Klaczany, G., Allen, K., Hershkowitz, D., Goldder, B. and Wolff, M. (2014) Evaluation of a Dentifrice Containing 8\% Arginine, Calcium Carbonate, and Sodium Monofluorophosphate to Repair AcidSoftened Enamel Using an Intra-Oral Remineralization Model. The Journal of Clinical Dentistry, 25, A14-A19.

[21] Wiegand, A., Köwing, L. and Attin, T. (2007) Impact of Brushing Force on Abrasion of Acid-Softened and Sound Enamel. Archives of Oral Biology, 52, 1043-1047. http://dx.doi.org/10.1016/j.archoralbio.2007.06.004

[22] Voronets, J. and Lussi, A. (2010) Thickness of Softened Human Enamel Removed by Toothbrush Abrasion: An in Vitro Study. Clinical Oral Investigations, 14, 251-256. http://dx.doi.org/10.1007/s00784-009-0288-y

[23] Shellis, R.P., Featherstone, J.D. and Lussi, A. (2014) Understanding the Chemistry of Dental Erosion. Monographs in Oral Science, 25, 163-179. http://dx.doi.org/10.1159/000359943

[24] Barbour, M.E. and Lussi, A. (2014) Erosion in Relation to Nutrition and the Environment. Monographs in Oral Science, 25, 143-154. http://dx.doi.org/10.1159/000359941

[25] Davis, W.B. and Winter, P.J. (1980) The Effect of Abrasion on Enamel and Dentine and Exposure to Dietary Acid. British Dental Journal, 148, 253-256. http://dx.doi.org/10.1038/sj.bdj.4804430

[26] Attin, T., Koidl, U., Buchalla, W., Schaller, H.G., Kielbassa, A.M. and Hellwig, E. (1997) Correlation of Microhardness and Wear in Differently Eroded Bovine Dental Enamel. Archives of Oral Biology, 42, 243-250. http://dx.doi.org/10.1016/0003-9969(06)00073-2

[27] Attin, T., Buchalla, W., Gollner, M. and Hellwig, E. (2000) Use of Variable Remineralization Periods to Improve the Abrasion Resistance of Previously Eroded Enamel. Caries Research, 34, 48-52. http://dx.doi.org/10.1159/000016569

[28] West, N.X. and Joiner, A. (2014) Enamel Mineral Loss. Journal of Dentistry, 42, S2-S11. http://dx.doi.org/10.1016/S0300-5712(14)50002-4

[29] Ganss, C., Schlueter, N., Friedrich, D. and Klimek, J. (2007) Efficacy of Waiting Periods and Topical Fluoride Treatment on Toothbrush Abrasion of Eroded Enamel in Situ. Caries Research, 41, 146-151. http://dx.doi.org/10.1159/000098049

[30] Hunter, M.L. and West, N.X. (2000) Mechanical Tooth Wear: The Role of Individual Toothbrush Variables and Toothpaste Abrasivity. In: Addy, M., Embery, G., Edgar, W.M. and Orchardson, R., Eds., Tooth Wear and Sensitivity, Martin Dunitz, London, 161-169. 